\title{
Delay-Dependent Guaranteed Cost Controller Design for Uncertain Neural Networks with Interval Time-Varying Delay
}

\author{
M. Rajchakit, ${ }^{1}$ P. Niamsup, ${ }^{2}$ T. Rojsiraphisal, ${ }^{2}$ and G. Rajchakit ${ }^{1}$ \\ ${ }^{1}$ Major of Mathematics and Statistics, Faculty of Science, Maejo University, \\ Chiang Mai 50290, Thailand \\ ${ }^{2}$ Department of Mathematics, Faculty of Science, Chiang Mai University, \\ Chiang Mai 50000, Thailand \\ Correspondence should be addressed to G. Rajchakit, mrajchakit@yahoo.com
}

Received 3 August 2012; Revised 24 September 2012; Accepted 25 September 2012

Academic Editor: Xiaodi Li

Copyright (c) 2012 M. Rajchakit et al. This is an open access article distributed under the Creative Commons Attribution License, which permits unrestricted use, distribution, and reproduction in any medium, provided the original work is properly cited.

This paper studies the problem of guaranteed cost control for a class of uncertain delayed neural networks. The time delay is a continuous function belonging to a given interval but not necessary to be differentiable. A cost function is considered as a nonlinear performance measure for the closed-loop system. The stabilizing controllers to be designed must satisfy some exponential stability constraints on the closed-loop poles. By constructing a set of augmented LyapunovKrasovskii functionals combined with Newton-Leibniz formula, a guaranteed cost controller is designed via memoryless state feedback control, and new sufficient conditions for the existence of the guaranteed cost state feedback for the system are given in terms of linear matrix inequalities (LMIs). Numerical examples are given to illustrate the effectiveness of the obtained result.

\section{Introduction}

The last few decades have witnessed the use of artificial neural networks (ANNs) in many real-world applications and have offered an attractive paradigm for a broad range of adaptive complex systems. In recent years, ANNs have enjoyed a great deal of success and have proven useful in wide variety pattern recognition feature-extraction tasks. Examples include optical character recognition, speech recognition, and adaptive control, to name a few. To keep the pace with the huge demand in diversified application areas, many different kinds of ANN architecture and learning types have been proposed to meet varying needs as robustness and stability. Stability and control of neural networks with time delay have attracted considerable 
attention in recent years [1-8]. In many practical systems, it is desirable to design neural networks which are not only asymptotically or exponentially stable but can also guarantee an adequate level of system performance. In the area of control, signal processing, pattern recognition, and image processing, delayed neural networks have many useful applications. Some of these applications require that the equilibrium points of the designed network be stable. In both biological and artificial neural systems, time delays due to integration and communication are ubiquitous and often become a source of instability. The time delays in electronic neural networks are usually time varying, and sometimes vary violently with respect to time due to the finite switching speed of amplifiers and faults in the electrical circuitry. Guaranteed cost control problem [9-12] has the advantage of providing an upper bound on a given system performance index and thus the system performance degradation incurred by the uncertainties or time delays is guaranteed to be less than this bound. The Lyapunov-Krasovskii functional technique has been among the popular and effective tool in the design of guaranteed cost controls for neural networks with time delay. Nevertheless, despite such diversity of results available, most existing work either assumed that the time delays are constant or differentiable [13-16]. Although, in some cases, delay-dependent guaranteed cost control for systems with time-varying delays was considered in $[12,13,15]$, the approach used there can not be applied to systems with interval, nondifferentiable timevarying delays. To the best of our knowledge, the guaranteed cost control and state feedback stabilization for uncertain neural networks with interval, non-differentiable time-varying delays have not been fully studied yet (see, e.g., [4-26] and the references therein), which are important in both theories and applications. This motivates our research.

In this paper, we investigate the guaranteed cost control for uncertain delayed neural networks problem. The novel features here are that the delayed neural network under consideration is with various globally Lipschitz continuous activation functions, and the time-varying delay function is interval, non-differentiable. A nonlinear cost function is considered as a performance measure for the closed-loop system. The stabilizing controllers to be designed must satisfy some exponential stability constraints on the closed-loop poles. Based on constructing a set of augmented Lyapunov-Krasovskii functionals combined with Newton-Leibniz formula, new delay-dependent criteria for guaranteed cost control via memoryless feedback control are established in terms of LMIs, which allow simultaneous computation of two bounds that characterize the exponential stability rate of the solution and can be easily determined by utilizing Matlabs LMI control toolbox.

The outline of the paper is as follows. Section 2 presents definitions and some wellknown technical propositions needed for the proof of the main result. LMI delay-dependent criteria for guaraneed cost control and a numerical examples showing the effectiveness of the result are presented in Section 3. The paper ends with conclusions and cited references.

\section{Preliminaries}

The following notation will be used in this paper. $\mathbb{R}^{+}$denotes the set of all real nonnegative numbers; $\mathbb{R}^{n}$ denotes the $n$-dimensional space with the scalar product $\langle x, y\rangle$ or $x^{T} y$ of two vectors $x, y$, and the vector norm $\|\cdot\| ; M^{n \times r}$ denotes the space of all matrices of $(n \times r)$ dimensions. $A^{T}$ denotes the transpose of matrix $A$; $A$ is symmetric if $A=A^{T}$; $I$ denotes the identity matrix; $\lambda(A)$ denotes the set of all eigenvalues of $A ; \lambda_{\max }(A)=\max \{\operatorname{Re} \lambda ; \lambda \in$ $\lambda(A)\} . x_{t}:=\{x(t+s): s \in[-h, 0]\},\left\|x_{t}\right\|=\sup _{s \in[-h, 0]}\|x(t+s)\| ; C^{1}\left([0, t], \mathbb{R}^{n}\right)$ denotes the set of all $\mathbb{R}^{n}$-valued continuously differentiable functions on $[0, t] ; L_{2}\left([0, t], \mathbb{R}^{m}\right)$ denotes the set of all the $\mathbb{R}^{m}$-valued square integrable functions on $[0, t]$. 
Matrix $A$ is called semipositive definite $(A \geq 0)$ if $\langle A x, x\rangle \geq 0$, for all $x \in \mathbb{R}^{n} ; A$ is positive definite $(A>0)$ if $\langle A x, x\rangle>0$ for all $x \neq 0 ; A>B$ means $A-B>0$. The notation $\operatorname{diag}\{\cdots\}$ stands for a block-diagonal matrix. The symmetric term in a matrix is denoted by $*$.

Consider the following uncertain neural networks with interval time-varying delay:

$$
\begin{gathered}
\dot{x}(t)=-(A+\Delta A(t)) x(t)+\left(W_{0}+\Delta W_{0}(t)\right) W_{0} f(x(t))+\left(W_{1}+\Delta W_{1}(t)\right) g(x(t-h(t))) \\
+B u(t), \quad t \geq 0, x(t)=\phi(t), t \in\left[-h_{1}, 0\right],
\end{gathered}
$$

where $x(t)=\left[x_{1}(t), x_{2}(t), \ldots, x_{n}(t)\right]^{T} \in \mathbb{R}^{n}$ is the state of the neural; $u(\cdot) \in L_{2}\left([0, t], \mathbb{R}^{m}\right)$ is the control; $n$ is the number of neurals, and

$$
\begin{gathered}
f(x(t))=\left[f_{1}\left(x_{1}(t)\right), f_{2}\left(x_{2}(t)\right), \ldots, f_{n}\left(x_{n}(t)\right)\right]^{T}, \\
g(x(t-h(t)))=\left[g_{1}\left(x_{1}(t-h(t))(t)\right), g_{2}\left(x_{2}(t-h(t))(t)\right), \ldots, g_{n}\left(x_{n}(t-h(t))\right)\right]^{T},
\end{gathered}
$$

are the activation functions; $A=\operatorname{diag}\left(\bar{a}_{1}, \bar{a}_{2}, \ldots, \bar{a}_{n}\right), \bar{a}_{i}>0$ represents the self-feedback term; $B \in R^{n \times m}$ is control input matrix; $W_{0}, W_{1}$ denote the connection weights, the discretely delayed connection weights and the distributively delayed connection weight, respectively; the time-varying uncertain matrices $\Delta A(t), \Delta W_{0}(t)$, and $\Delta W_{1}(t)$ are defined by

$$
\Delta A(t)=E_{a} F_{a}(t) H_{a}, \quad \Delta W_{0}(t)=E_{w_{0}} F_{w_{0}}(t) H_{w_{0}}, \quad \Delta W_{1}(t)=E_{w_{1}} F_{w_{1}}(t) H_{w_{1}},
$$

where $E_{a}, E_{w_{0}}, E_{w_{1}}, H_{a}, H_{w_{0}}$, and $H_{w_{1}}$ are known constant real matrices with appropriate dimensions. $F_{a}(t), F_{w_{0}}(t)$, and $F_{w_{1}}(t)$ are unknown uncertain matrices satisfying

$$
F_{a}^{T}(t) F_{a}(t) \leq I, \quad F_{w_{0}}^{T}(t) F_{w_{0}}(t) \leq I, \quad F_{w_{1}}^{T}(t) F_{w_{1}}(t) \leq I, \quad t \in R^{+} .
$$

The time-varying delay function $h(t)$ satisfies the condition

$$
0 \leq h_{0} \leq h(t) \leq h_{1} .
$$

The initial functions $\phi(t) \in C^{1}\left(\left[-h_{1}, 0\right], R^{n}\right)$, with the norm

$$
\|\phi\|=\sup _{t \in\left[-h_{1}, 0\right]} \sqrt{\|\phi(t)\|^{2}+\|\dot{\phi}(t)\|^{2}} .
$$

In this paper we consider various activation functions and assume that the activation functions $f(\cdot), g(\cdot)$ are Lipschitzian with the Lipschitz constants $f_{i}, e_{i}>0$ :

$$
\begin{aligned}
& \left|f_{i}\left(\xi_{1}\right)-f_{i}\left(\xi_{2}\right)\right| \leq f_{i}\left|\xi_{1}-\xi_{2}\right|, \quad i=1,2, \ldots, n, \forall \xi_{1}, \xi_{2} \in \mathbb{R}, \\
& \left|g_{i}\left(\xi_{1}\right)-g_{i}\left(\xi_{2}\right)\right| \leq e_{i}\left|\xi_{1}-\xi_{2}\right|, \quad i=1,2, \ldots, n, \forall \xi_{1}, \xi_{2} \in \mathbb{R} .
\end{aligned}
$$


The performance index associated with the system (2.1) is the following function:

$$
J=\int_{0}^{\infty} f^{0}(t, x(t), x(t-h(t)), u(t)) d t
$$

where $f^{0}(t, x(t), x(t-h(t)), u(t)): R^{+} \times R^{n} \times R^{n} \times R^{m} \rightarrow R^{+}$is a nonlinear cost function that satisfies

$$
\exists Q_{1}, Q_{2}, R: f^{0}(t, x, y, u) \leq\left\langle Q_{1} x, x\right\rangle+\left\langle Q_{2} y, y\right\rangle+\langle R u, u\rangle,
$$

for all $(t, x, u) \in R^{+} \times R^{n} \times R^{m}$ and $Q_{1}, Q_{2} \in R^{n \times n}, R \in R^{m \times m}$ are given symmetric positive definite matrices. The objective of this paper is to design a memoryless state feedback controller $u(t)=K x(t)$ for system (2.1) and the cost function (2.8) such that the resulting closed-loop system

$$
\begin{aligned}
\dot{x}(t)= & -\left[\left(A+E_{a} F_{a}(t) H_{a}\right)-B K\right] x(t)+\left(W_{0}+E_{w_{0}} F_{w_{0}}(t) H_{w_{0}}\right) f(x(t)) \\
& +\left(W_{1}+E_{w_{1}} F_{w_{1}}(t) H_{w_{1}}\right) g(x(t-h(t)))
\end{aligned}
$$

is exponentially stable and the closed-loop value of the cost function (2.10) is minimized.

Definition 2.1. Given $\alpha>0$. The zero solution of closed-loop system (2.8) is $\alpha$-exponentially stabilizable if there exists a positive number $N>0$ such that every solution $x(t, \phi)$ satisfies the following condition:

$$
\|x(t, \phi)\| \leq N e^{-\alpha t}\|\phi\|, \quad \forall t \geq 0 .
$$

Definition 2.2. Consider the control system (2.1). If there exists a memoryless state feedback control law $u^{*}(t)=K x(t)$ and a positive number $J^{*}$ such that the zero solution of the closedloop system (2.10) is exponentially stable and the cost function (2.8) satisfies $J \leq J^{*}$, then the value $J^{*}$ is a guaranteed constant and $u^{*}(t)$ is a guaranteed cost control law of the system and its corresponding cost function.

We introduce the following technical well-known propositions, which will be used in the proof of our results.

Proposition 2.3 (Schur complement lemma [27]). Given constant matrices $X, Y$, and $Z$ with appropriate dimensions satisfying $X=X^{T}, Y=Y^{T}>0$, then $X+Z^{T} Y^{-1} Z<0$ if and only if

$$
\left(\begin{array}{cc}
X & Z^{T} \\
Z & -Y
\end{array}\right)<0
$$


Proposition 2.4 (integral matrix inequality [28]). For any symmetric positive definite matrix $M>$ 0, scalar $\gamma>0$ and vector function $\omega:[0, \gamma] \rightarrow R^{n}$ such that the integrations concerned are well defined, the following inequality holds

$$
\left(\int_{0}^{\gamma} \omega(s) d s\right)^{T} M\left(\int_{0}^{\gamma} \omega(s) d s\right) \leq r\left(\int_{0}^{\gamma} \omega^{T}(s) M \omega(s) d s\right)
$$

\section{Design of Guaranteed Cost Controller}

In this section, we give a design of memoryless guaranteed feedback cost control for uncertain neural networks (2.1). Let us set

$$
\begin{aligned}
& W_{11}=-P A^{T}-A P-2 \alpha P+0.25 B R B^{T}+\sum_{i=0}^{1} G_{i}+2 \epsilon_{1} E_{a}^{T} E_{a}+6 \epsilon_{1}^{-1} P H_{a}^{T} H_{a} P \\
& +4 \epsilon_{2}^{-1} \mathrm{PFH}_{w_{0}}^{T} H_{w_{0}} F P, \\
& W_{12}=P+A P-0.5 B B^{T}, \quad W_{13}=e^{-2 \alpha h_{0}} H_{0}+0.5 B B^{T}+A P, \\
& W_{14}=e^{-2 \alpha h_{1}} H_{1}+0.5 B B^{T}+A P, \quad W_{15}=P+0.5 B B^{T}+A P, \\
& W_{22}=\sum_{i=0}^{1} W_{i} D_{i} W_{i}^{T}+\sum_{i=0}^{1} h_{i}^{2} H_{i}+\left(h_{1}-h_{0}\right) U-2 P-B B^{T}+\epsilon_{1} E_{a}^{T} E_{a}+\epsilon_{2} E_{w_{0}}^{T} E_{w_{0}}+\epsilon_{3} E_{w_{1}}^{T} E_{w_{1}}, \\
& W_{23}=P, \quad W_{24}=P, \quad W_{25}=P, \\
& W_{33}=-e^{-2 \alpha h_{0}} G_{0}-e^{-2 \alpha h_{0}} H_{0}-e^{-2 \alpha h_{1}} U+\sum_{i=0}^{1} W_{i} D_{i} W_{i}^{T}+\epsilon_{1} E_{a}^{T} E_{a}+\epsilon_{2} E_{w_{0}}^{T} E_{w_{0}}+\epsilon_{3} E_{w_{1}}^{T} E_{w_{1}}, \\
& W_{34}=0, \quad W_{35}=e^{-2 \alpha h_{1}} U, \\
& W_{44}=\sum_{i=0}^{1} W_{i} D_{i} W_{i}^{T}-e^{-2 \alpha h_{1}} U-e^{-2 \alpha h_{1}} G_{1}-e^{-2 \alpha h_{1}} H_{1}+\epsilon_{1} E_{a}^{T} E_{a}+\epsilon_{2} E_{w_{0}}^{T} E_{w_{0}}+\epsilon_{3} E_{w_{1}}^{T} E_{w_{1}}, \\
& W_{45}=e^{-2 \alpha h_{1}} U, \\
& W_{55}=-e^{-2 \alpha h_{1}} U+W_{0} D_{0} W_{0}^{T}+4 \epsilon_{3}^{-1} P E H_{w_{1}}^{T} H_{w_{1}} E P+\epsilon_{1} E_{a}^{T} E_{a}+\epsilon_{2} E_{w_{0}}^{T} E_{w_{0}}+\epsilon_{3} E_{w_{1}}^{T} E_{w_{1}}, \\
& E=\operatorname{diag}\left\{e_{i}, i=1, \ldots, n\right\}, \quad F=\operatorname{diag}\left\{f_{i}, i=1, \ldots, n\right\}, \\
& \lambda_{1}=\lambda_{\min }\left(P^{-1}\right) \\
& \lambda_{2}=\lambda_{\max }\left(P^{-1}\right)+h_{0} \lambda_{\max }\left[P^{-1}\left(\sum_{i=0}^{1} G_{i}\right) P^{-1}\right] \\
& +h_{1}^{2} \lambda_{\max }\left[P^{-1}\left(\sum_{i=0}^{1} H_{i}\right) P^{-1}\right]+\left(h_{1}-h_{0}\right) \lambda_{\max }\left(P^{-1} U P^{-1}\right) \text {. }
\end{aligned}
$$


Theorem 3.1. Consider control system (2.1) and the cost function (2.8). If there exist symmetric positive definite matrices $P, U, G_{0}, G_{1}, H_{0}$, and $H_{1}$, and diagonal positive definite matrices $D_{i}, i=$ 0,1 , and $\epsilon_{i}>0, i=1,2,3$ satisfying the following LMIs

$$
\begin{aligned}
& \varepsilon=\left[\begin{array}{ccccc}
W_{11} & W_{12} & W_{13} & W_{14} & W_{15} \\
* & W_{22} & W_{23} & W_{24} & W_{25} \\
* & * & W_{33} & W_{34} & W_{35} \\
* & * & * & W_{44} & W_{45} \\
* & * & * & * & W_{55}
\end{array}\right]<0 \\
& \mathcal{S}_{1}=\left[\begin{array}{ccc}
-P A-A^{T} P-\sum_{i=0}^{1} e^{-2 \alpha h_{i}} H_{i} & 2 P F & P Q_{1} \\
* & -D_{0} & 0 \\
* & * & -Q_{1}^{-1}
\end{array}\right]<0, \\
& S_{2}=\left[\begin{array}{ccc}
W_{1} D_{1} W_{1}^{T}-e^{-2 \alpha h_{1}} U & 2 P E & P Q_{2} \\
* & -D_{1} & 0 \\
* & * & -Q_{2}^{-1}
\end{array}\right]<0 \text {, }
\end{aligned}
$$

then

$$
u(t)=-\frac{1}{2} B^{T} P^{-1} x(t), \quad t \geq 0
$$

is a guaranteed cost control and the guaranteed cost value is given by

$$
J^{*}=\lambda_{2}\|\phi\|^{2}
$$

Moreover, the solution $x(t, \phi)$ of the system satisfies

$$
\|x(t, \phi)\| \leq \sqrt{\frac{\lambda_{2}}{\lambda_{1}}} e^{-\alpha t}\|\phi\|, \quad \forall t \geq 0 .
$$

Proof. Let $Y=P^{-1}, y(t)=Y x(t)$. Using the feedback control (2.8) we consider the following Lyapunov-Krasovskii functional:

$$
\begin{aligned}
V\left(t, x_{t}\right) & =\sum_{i=1}^{6} V_{i}\left(t, x_{t}\right), \\
V_{1} & =x^{T}(t) Y x(t), \\
V_{2} & =\int_{t-h_{0}}^{t} e^{2 \alpha(s-t)} x^{T}(s) Y G_{0} Y x(s) d s, \\
V_{3} & =\int_{t-h_{1}}^{t} e^{2 \alpha(s-t)} x^{T}(s) Y G_{1} Y x(s) d s,
\end{aligned}
$$


Abstract and Applied Analysis

$$
\begin{aligned}
& V_{4}=h_{0} \int_{-h_{0}}^{0} \int_{t+s}^{t} e^{2 \alpha(\tau-t)} \dot{x}^{T}(\tau) Y H_{0} Y \dot{x}(\tau) d \tau d s, \\
& V_{5}=h_{1} \int_{-h_{1}}^{0} \int_{t+s}^{t} e^{2 \alpha(\tau-t)} \dot{x}^{T}(\tau) Y H_{1} Y \dot{x}(\tau) d \tau d s, \\
& V_{6}=\left(h_{1}-h_{0}\right) \int_{t-h_{1}}^{t-h_{0}} \int_{t+s}^{t} e^{2 \alpha(\tau-t)} \dot{x}^{T}(\tau) Y U Y \dot{x}(\tau) d \tau d s .
\end{aligned}
$$

It is easy to check that

$$
\lambda_{1}\|x(t)\|^{2} \leq V\left(t, x_{t}\right) \leq \lambda_{2}\left\|x_{t}\right\|^{2}, \quad \forall t \geq 0 .
$$

Taking the derivative of $V_{1}$ we have

$$
\begin{aligned}
\dot{V}_{1}= & 2 x^{T}(t) Y \dot{x}(t) \\
= & y^{T}(t)\left[-P\left(A+E_{a} F_{a}(t) H_{a}\right)^{T}-\left(A+E_{a} F_{a}(t) H_{a}\right) P\right] y(t)-y^{T}(t) B B^{T} y(t) \\
& +2 y^{T}(t)\left(W_{0}+E_{w_{0}} F_{w_{0}}(t) H_{w_{0}}\right) f(\cdot) y(t)+2 y^{T}(t)\left(W_{1}+E_{w_{1}} F_{w_{1}}(t) H_{w_{1}}\right) g(\cdot) y(t), \\
\dot{V}_{2}= & y^{T}(t) G_{0} y(t)-e^{-2 \alpha h_{0}} y^{T}\left(t-h_{0}\right) G_{0} y\left(t-h_{0}\right)-2 \alpha V_{2}, \\
\dot{V}_{3}= & y^{T}(t) G_{1} y(t)-e^{-2 \alpha h_{1}} y^{T}\left(t-h_{1}\right) G_{1} y\left(t-h_{1}\right)-2 \alpha V_{3}, \\
\dot{V}_{4}= & h_{0}^{2} \dot{y}^{T}(t) H_{0} \dot{y}(t)-h_{1} e^{-2 \alpha h_{0}} \int_{t-h_{0}}^{t} \dot{x}^{T}(s) H_{0} \dot{x}(s) d s-2 \alpha V_{4}, \\
\dot{V}_{5}= & h_{1}^{2} \dot{y}^{T}(t) H_{1} \dot{y}(t)-h_{1} e^{-2 \alpha h_{1}} \int_{t-h_{1}}^{t} \dot{y}^{T}(s) H_{1} \dot{y}(s) d s-2 \alpha V_{4}, \\
\dot{V}_{6}= & \left(h_{1}-h_{0}\right)^{2} \dot{y}^{T}(t) U \dot{y}(t)-\left(h_{1}-h_{0}\right) e^{-2 \alpha h_{1}} \int_{t-h_{1}}^{t-h_{0}} \dot{y}^{T}(s) U \dot{y}(s) d s-2 \alpha V_{6},
\end{aligned}
$$

Applying Proposition 2.4 and the Leibniz-Newton formula

$$
\int_{s}^{t} \dot{y}(\tau) d \tau=y(t)-y(s)
$$


We have for $j=1,2, i=0,1$

$$
\begin{aligned}
-h_{i} \int_{t-h_{i}}^{t} \dot{y}^{T}(s) H_{j} \dot{y}(s) d s \leq & -\left[\int_{t-h_{i}}^{t} \dot{y}(s) d s\right]^{T} H_{j}\left[\int_{t-h_{i}}^{t} \dot{y}(s) d s\right] \\
\leq & -[y(t)-y(t-h(t))]^{T} H_{j}[y(t)-y(t-h(t))] \\
= & -y^{T}(t) H_{i} y(t)+2 x^{T}(t) H_{j} y(t-h(t)) \\
& -y^{T}\left(t-h_{i}\right) H_{j} y\left(t-h_{i}\right) .
\end{aligned}
$$

Note that

$$
\int_{t-h_{1}}^{t-h_{0}} \dot{y}^{T}(s) U \dot{y}(s) d s=\int_{t-h_{1}}^{t-h(t)} \dot{y}^{T}(s) U \dot{y}(s) d s+\int_{t-h(t)}^{t-h_{0}} \dot{y}^{T}(s) U \dot{y}(s) d s .
$$

Applying Proposition 2.4 gives

$$
\begin{aligned}
{\left[h_{1}-h(t)\right] \int_{t-h_{1}}^{t-h(t)} \dot{y}^{T}(s) U \dot{y}(s) d s } & \geq\left[\int_{t-h_{1}}^{t-h(t)} \dot{y}(s) d s\right]^{T} U\left[\int_{t-h_{1}}^{t-h(t)} \dot{y}(s) d s\right] \\
& \geq\left[y\left(t-h(t)-y\left(t-h_{1}\right)\right)\right]^{T} U\left[y\left(t-h(t)-y\left(t-h_{1}\right)\right)\right] .
\end{aligned}
$$

Since $h_{1}-h(t) \leq h_{1}-h_{0}$, we have

$$
\left[h_{1}-h_{0}\right] \int_{t-h_{1}}^{t-h(t)} \dot{y}^{T}(s) U \dot{y}(s) d s \geq\left[y\left(t-h(t)-y\left(t-h_{1}\right)\right)\right]^{T} U\left[y\left(t-h(t)-y\left(t-h_{1}\right)\right)\right],
$$

then

$$
-\left[h_{1}-h_{0}\right] \int_{t-h_{1}}^{t-h(t)} \dot{y}^{T}(s) U \dot{y}(s) d s \leq-\left[y\left(t-h(t)-y\left(t-h_{1}\right)\right)\right]^{T} U\left[y\left(t-h(t)-y\left(t-h_{1}\right)\right)\right]
$$

Similarly, we have

$$
-\left(h_{1}-h_{0}\right) \int_{t-h(t)}^{t-h_{0}} \dot{y}^{T}(s) U \dot{y}(s) d s \leq-\left[y\left(t-h_{0}\right)-y(t-h(t))\right]^{T} U\left[y\left(t-h_{0}\right)-y(t-h(t))\right] .
$$


Then, we have

$$
\begin{aligned}
\dot{V}(\cdot)+2 \alpha V(\cdot) \leq & y^{T}(t)\left[-P\left(A+E_{a} F_{a}(t) H_{a}\right)^{T}-\left(A+E_{a} F_{a}(t) H_{a}\right) P\right] y(t)-y^{T}(t) B B^{T} y(t) \\
& +2 y^{T}(t)\left(W_{0}+E_{w_{0}} F_{w_{0}}(t) H_{w_{0}}\right) f(\cdot)+2 y^{T}(t)\left(W_{1}+E_{w_{1}} F_{w_{1}}(t) H_{w_{1}}\right) g(\cdot) \\
& +y^{T}(t)\left(\sum_{i=0}^{1} G_{i}\right) y(t)+2 \alpha\langle P y(t), y(t)\rangle \\
& +\dot{y}^{T}(t)\left(\sum_{i=0}^{1} h_{i}^{2} H_{i}\right) \dot{y}(t)+\left(h_{1}-h_{0}\right) \dot{y}^{T}(t) U \dot{y}(t) \\
& -\sum_{i=0}^{1} e^{-2 \alpha h_{i}} y^{T}\left(t-h_{i}\right) G_{i} y\left(t-h_{i}\right) \\
& -e^{-2 \alpha h_{0}}\left[y(t)-y\left(t-h_{0}\right)\right]^{T} H_{0}\left[y(t)-y\left(t-h_{0}\right)\right] \\
& -e^{-2 \alpha h_{1}}\left[y(t)-y\left(t-h_{1}\right)\right]^{T} H_{1}\left[y(t)-y\left(t-h_{1}\right)\right] \\
& -e^{-2 \alpha h_{1}}\left[y(t-h(t))-y\left(t-h_{1}\right)\right]^{T} U\left[y(t-h(t))-y\left(t-h_{1}\right)\right] \\
& -e^{-2 \alpha h_{1}}\left[y\left(t-h_{0}\right)-y(t-h(t))\right]^{T} U\left[y\left(t-h_{0}\right)-y(t-h(t))\right] .
\end{aligned}
$$

Using (2.8)

$$
\begin{aligned}
P \dot{y}(t) & +\left(A+E_{a} F_{a}(t) H_{a}\right) P y(t)-\left(W_{0}+E_{w_{0}} F_{w_{0}}(t) H_{w_{0}}\right) f(\cdot)-\left(W_{1}+E_{w_{1}} F_{w_{1}}(t) H_{w_{1}}\right) g(\cdot) \\
& +0.5 B B^{T} y(t)=0
\end{aligned}
$$

and multiplying both sides with $\left[2 y(t),-2 \dot{y}(t), 2 y\left(t-h_{0}\right), 2 y\left(t-h_{1}\right), 2 y(t-h(t))\right]^{T}$, we have

$$
\begin{aligned}
& 2 y^{T}(t) P \dot{y}(t)+2 y^{T}(t)\left(A+E_{a} F_{a}(t) H_{a}\right) P y(t)-2 y^{T}(t)\left(W_{0}+E_{w_{0}} F_{w_{0}}(t) H_{w_{0}}\right) f(\cdot) \\
& \quad-2 y^{T}(t)\left(W_{1}+E_{w_{1}} F_{w_{1}}(t) H_{w_{1}}\right) g(\cdot)+y^{T}(t) B B^{T} y(t)=0, \\
& -2 \dot{y}^{T}(t) P \dot{y}(t)-2 \dot{y}^{T}(t)\left(A+E_{a} F_{a}(t) H_{a}\right) P y(t)+2 \dot{y}^{T}(t)\left(W_{0}+E_{w_{0}} F_{w_{0}}(t) H_{w_{0}}\right) f(\cdot) \\
& \quad+2 \dot{y}^{T}(t)\left(W_{1}+E_{w_{1}} F_{w_{1}}(t) H_{w_{1}}\right) g(\cdot)-\dot{y}^{T}(t) B B^{T} y(t)=0, \\
& 2 y^{T}\left(t-h_{0}\right) P \dot{y}(t)+2 y^{T}\left(t-h_{0}\right)\left(A+E_{a} F_{a}(t) H_{a}\right) P y(t)-2 y^{T}\left(t-h_{0}\right)\left(W_{0}+E_{w_{0}} F_{w_{0}}(t) H_{w_{0}}\right) \\
& \quad \times f(\cdot)-2 y^{T}\left(t-h_{0}\right)\left(W_{1}+E_{w_{1}} F_{w_{1}}(t) H_{w_{1}}\right) g(\cdot)+y^{T}\left(t-h_{0}\right) B B^{T} y(t)=0,
\end{aligned}
$$




$$
\begin{aligned}
& 2 y^{T}\left(t-h_{1}\right) P \dot{y}(t)+2 y^{T}\left(t-h_{1}\right)\left(A+E_{a} F_{a}(t) H_{a}\right) P y(t)-2 y^{T}\left(t-h_{1}\right)\left(W_{0}+E_{w_{0}} F_{w_{0}}(t) H_{w_{0}}\right) \\
& \quad \times f(\cdot)-2 y^{T}\left(t-h_{1}\right)\left(W_{1}+E_{w_{1}} F_{w_{1}}(t) H_{w_{1}}\right) g(\cdot)+y^{T}\left(t-h_{1}\right) B B^{T} y(t)=0 \\
& 2 y^{T}(t-h(t)) P \dot{y}(t)+2 y^{T}(t-h(t))\left(A+E_{a} F_{a}(t) H_{a}\right) P y(t)-2 y^{T}(t-h(t)) \\
& \quad \times\left(W_{0}+E_{w_{0}} F_{w_{0}}(t) H_{w_{0}}\right) f(\cdot)-2 y^{T}(t-h(t))\left(W_{1}+E_{w_{1}} F_{w_{1}}(t) H_{w_{1}}\right) g(\cdot) \\
& \quad+y^{T}(t-h(t)) B B^{T} y(t)=0 .
\end{aligned}
$$

Adding all the zero items of (3.20) and $f^{0}(t, x(t), x(t-h(t)), u(t))-f^{0}(t, x(t), x(t-h(t)), u(t))=$ 0 , respectively, into (3.18) and using the condition (2.7) for the following estimations:

$$
\begin{aligned}
f^{0}(t, x(t), x(t-h(t)), u(t)) \leq & \left\langle Q_{1} x(t), x(t)\right\rangle+\left\langle Q_{2} x(t-h(t)), x(t-h(t))\right\rangle+\langle R u(t), u(t)\rangle \\
= & \left\langle P Q_{1} P y(t), y(t)\right\rangle+\left\langle P Q_{2} P y(t-h(t)), y(t-h(t))\right\rangle \\
& +0.25\left\langle B R B^{T} y(t), y(t)\right\rangle, \\
2\left\langle W_{0} f(x), y\right\rangle \leq & \left\langle W_{0} D_{0} W_{0}^{T} y, y\right\rangle+\left\langle D_{0}^{-1} f(x), f(x)\right\rangle, \\
2\left\langle W_{1} g(z), y\right\rangle \leq & \left\langle W_{1} D_{1} W_{1}^{T} y, y\right\rangle+\left\langle D_{1}^{-1} g(z), g(z)\right\rangle, \\
2\left\langle D_{0}^{-1} f(x), f(x)\right\rangle \leq & \left\langle F D_{0}^{-1} F x, x\right\rangle, \\
2\left\langle D_{1}^{-1} g(z), g(z)\right\rangle \leq & \left\langle E D_{1}^{-1} E z, z\right\rangle, \\
2\left\langle E_{a} F_{a}(t) H_{a} P y, y\right\rangle \leq & \left\langle\epsilon_{1} E_{a}^{T} E_{a} y, y\right\rangle+\left\langle\epsilon_{1}^{-1} P H_{a}^{T} H_{a} P y, y\right\rangle, \quad \epsilon_{1}>0, \\
2\left\langle E_{w_{0}} F_{w_{0}}(t) H_{w_{0}} P f(x), y\right\rangle \leq & \left\langle\epsilon_{2} E_{w_{0}}^{T} E_{w_{0}} y, y\right\rangle+\left\langle\epsilon_{2}^{-1} P D_{0} H_{w_{0}}^{T} H_{w_{0}} D_{0} P y, y\right\rangle, \quad \epsilon_{2}>0, \\
2\left\langle E_{w_{1}} F_{w_{1}}(\mathrm{t}) H_{w_{1}} P g(z), y\right\rangle \leq & \left\langle\epsilon_{3} E_{w_{1}}^{T} E_{w_{1}} y, y\right\rangle+\left\langle\epsilon_{3}^{-1} P D_{1} H_{w_{1}}^{T} H_{w_{1}} D_{1} P z, z\right\rangle, \quad \epsilon_{3}>0,
\end{aligned}
$$

we obtain

$$
\begin{aligned}
\dot{V}(\cdot)+2 \alpha V(\cdot) \leq & \zeta^{T}(t) \mathcal{E} \zeta(t)+y^{T}(t) S_{1} y(t)+y^{T}(t-h(t)) S_{2} y(t-h(t)) \\
& -f^{0}(t, x(t), x(t-h(t)), u(t)),
\end{aligned}
$$


where $\zeta(t)=\left[y(t), \dot{y}(t), y\left(t-h_{0}\right), y\left(t-h_{1}\right), y(t-h(t))\right]$, and

$$
\begin{gathered}
\varepsilon=\left[\begin{array}{ccccc}
W_{11} & W_{12} & W_{13} & W_{14} & W_{15} \\
* & W_{22} & W_{23} & W_{24} & W_{25} \\
* & * & W_{33} & W_{34} & W_{35} \\
* & * & * & W_{44} & W_{45} \\
* & * & * & * & W_{55}
\end{array}\right], \\
S_{1}=-P A-A^{T} P-\sum_{i=0}^{1} e^{-2 \alpha h_{i}} H_{i}+4 P F D_{0}^{-1} F P+P Q_{1} P, \\
S_{2}=W_{1} D_{1} W_{1}^{T}-e^{-2 \alpha h_{2}} U+4 P E D_{1}^{-1} E P+P Q_{2} P .
\end{gathered}
$$

Note that by the Schur complement lemma, Proposition 2.3, the conditions $S_{1}<0$ and $S_{2}<0$ are equivalent to the conditions (3.3) and (3.4), respectively. Therefore, by condition (3.2), (3.3), and (3.4), we obtain from (3.22) that

$$
\dot{V}\left(t, x_{t}\right) \leq-2 \alpha V\left(t, x_{t}\right), \quad \forall t \geq 0
$$

Integrating both sides of (3.24) from 0 to $t$, we obtain

$$
V\left(t, x_{t}\right) \leq V(\phi) e^{-2 \alpha t}, \quad \forall t \geq 0
$$

Furthermore, taking condition (3.9) into account, we have

$$
\lambda_{1}\|x(t, \phi)\|^{2} \leq V\left(x_{t}\right) \leq V(\phi) e^{-2 \alpha t} \leq \lambda_{2} e^{-2 \alpha t}\|\phi\|^{2},
$$

then

$$
\|x(t, \phi)\| \leq \sqrt{\frac{\lambda_{2}}{\lambda_{1}}} e^{-\alpha t}\|\phi\|, \quad t \geq 0
$$

which concludes the exponential stability of the closed-loop system (2.8). To prove the optimal level of the cost function (2.4), we derive from (3.22) and (3.2)-(3.4) that

$$
\dot{V}\left(t, z_{t}\right) \leq-f^{0}(t, x(t), x(t-h(t)), u(t)), \quad t \geq 0 .
$$

Integrating both sides of (3.28) from 0 to $t$ leads to

$$
\int_{0}^{t} f^{0}(t, x(t), x(t-h(t)), u(t)) d t \leq V\left(0, z_{0}\right)-V\left(t, z_{t}\right) \leq V\left(0, z_{0}\right)
$$


dute to $V\left(t, z_{t}\right) \geq 0$. Hence, letting $t \rightarrow+\infty$, we have

$$
J=\int_{0}^{\infty} f^{0}(t, x(t), x(t-h(t)), u(t)) d t \leq V\left(0, z_{0}\right) \leq \lambda_{2}\|\phi\|^{2}=J^{*} .
$$

This completes the proof of the theorem.

Remark 3.2. Note that $h(t)$ is non-differentiable and interval time-varying delay; therefore, the stability criteria proposed in $[5-8,12,15-26]$ are not applicable to this system.

Example 3.3. Consider the uncertain neural networks with interval time-varying delays (2.1), where

$$
\begin{gathered}
A=\left[\begin{array}{cc}
0.1 & 0 \\
0 & 0.3
\end{array}\right], \quad W_{0}=\left[\begin{array}{ll}
0.1 & 0.1 \\
0.2 & 0.3
\end{array}\right], \quad W_{1}=\left[\begin{array}{ll}
0.2 & 0.2 \\
0.1 & 0.4
\end{array}\right], \quad B=\left[\begin{array}{l}
0.1 \\
0.2
\end{array}\right], \\
E=\left[\begin{array}{cc}
0.3 & 0 \\
0 & 0.4
\end{array}\right], \quad F=\left[\begin{array}{cc}
0.2 & 0 \\
0 & 0.3
\end{array}\right], \quad Q_{1}=\left[\begin{array}{ll}
0.2 & 0.1 \\
0.1 & 0.4
\end{array}\right], \quad Q_{2}=\left[\begin{array}{ll}
0.3 & 0.2 \\
0.2 & 0.5
\end{array}\right], \\
R=\left[\begin{array}{cc}
0.1 & 0.1 \\
0.1 & 0.3
\end{array}\right], \quad E_{a}=\left[\begin{array}{cc}
0.1 & 0.1 \\
0.1 & 0.3
\end{array}\right], \quad E_{w_{0}}=\left[\begin{array}{ll}
0.1 & 0.1 \\
0.1 & 0.2
\end{array}\right], \quad E_{w_{1}}=\left[\begin{array}{ll}
0.2 & 0.1 \\
0.1 & 0.3
\end{array}\right], \\
H_{a}=\left[\begin{array}{ll}
0.3 & 0.2 \\
0.2 & 0.2
\end{array}\right], \quad H_{w_{0}}=\left[\begin{array}{ll}
0.2 & 0.1 \\
0.1 & 0.2
\end{array}\right], \quad H_{w_{1}}=\left[\begin{array}{ll}
0.3 & 0.1 \\
0.1 & 0.3
\end{array}\right], \\
\left.h(t)=0.1+1.3 \sin ^{2} t \quad \text { if } t \in \supset=\bigcup \begin{array}{l}
\bigcup \\
h(t)=0
\end{array} \quad \text { if } t \in R^{+} \backslash \supset .(2 k+1) \pi\right]
\end{gathered}
$$

Note that $h(t)$ is non-differentiable; therefore, the stability criteria proposed in [4-8, 12, 15-26] are not applicable to this system. Given $\alpha=0.1, h_{0}=0.1$, and $h_{1}=1.4$, by using the Matlab LMI toolbox, we can solve for $P, U, G_{0}, G_{1}, H_{0}, H_{1}, D_{0}$, and $D_{1}$ which satisfy the conditions (3.2)-(3.4) in Theorem 3.1. A set of solutions are $\epsilon_{1}=0.0017, \epsilon_{2}=0.0013, \epsilon_{3}=0.0012$,

$$
\begin{array}{cc}
P=\left[\begin{array}{cc}
1.1578 & -0.1128 \\
-0.1128 & 1.0597
\end{array}\right], & U=\left[\begin{array}{cc}
2.3269 & -0.3820 \\
-0.3820 & 2.6681
\end{array}\right], \\
G_{0}=\left[\begin{array}{ll}
1.4596 & 0.1397 \\
0.1397 & 1.2369
\end{array}\right], & G_{1}=\left[\begin{array}{ll}
2.2694 & 0.8114 \\
0.8114 & 1.0125
\end{array}\right], \\
H_{0}=\left[\begin{array}{cc}
0.6455 & 0.0452 \\
0.0452 & 0.5104
\end{array}\right], & H_{1}=\left[\begin{array}{ll}
0.3005 & 0.0233 \\
0.0233 & 0.2306
\end{array}\right], \\
D_{0}=\left[\begin{array}{cc}
0.0011 & 0 \\
0 & 0.0011
\end{array}\right], & D_{1}=\left[\begin{array}{cc}
0.7809 & 0 \\
0 & 0.7809
\end{array}\right] .
\end{array}
$$

Then

$$
u(t)=-0.2292 x_{1}(t)-0.1816 x_{2}(t), \quad t \geq 0
$$




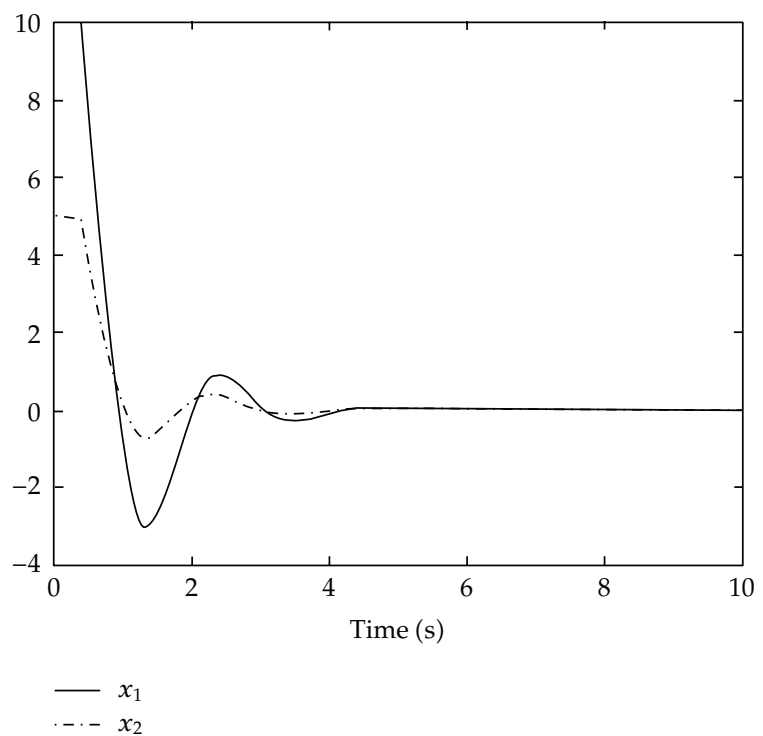

Figure 1: The simulation of the solutions $x_{1}(t)$ and $x_{2}(t)$ with the initial condition $\phi\left(t[105]^{T}, t \in[-0.4,0]\right.$.

is a guaranteed cost control law and the cost given by

$$
J^{*}=5.4631\|\phi\|^{2}
$$

Moreover, the solution $x(t, \phi)$ of the system satisfies

$$
\|x(t, \phi)\| \leq 3.6984 e^{-0.1 t}\|\phi\|, \quad \forall t \geq 0 .
$$

The exponential convergence dynamics of the network (2.1) are shown in Figure 1.

Example 3.4. Consider the uncertain neural networks with interval time-varying delays (2.1), where

$$
\begin{gathered}
A=\left[\begin{array}{ll}
1 & 0 \\
0 & 2
\end{array}\right], \quad W_{0}=\left[\begin{array}{ll}
1 & 1 \\
2 & 3
\end{array}\right], \quad W_{1}=\left[\begin{array}{ll}
2 & 2 \\
1 & 4
\end{array}\right], \quad B=\left[\begin{array}{l}
1 \\
2
\end{array}\right], \\
E=\left[\begin{array}{ll}
3 & 0 \\
0 & 4
\end{array}\right], \quad F=\left[\begin{array}{ll}
2 & 0 \\
0 & 3
\end{array}\right], \quad Q_{1}=\left[\begin{array}{ll}
2 & 1 \\
1 & 4
\end{array}\right], \quad Q_{2}=\left[\begin{array}{ll}
3 & 2 \\
2 & 5
\end{array}\right], \\
R=\left[\begin{array}{ll}
1 & 1 \\
1 & 3
\end{array}\right], \quad E_{a}=\left[\begin{array}{ll}
1 & 1 \\
1 & 3
\end{array}\right], \quad E_{w_{0}}=\left[\begin{array}{ll}
1 & 1 \\
1 & 2
\end{array}\right], \quad E_{w_{1}}=\left[\begin{array}{ll}
2 & 1 \\
1 & 3
\end{array}\right], \\
H_{a}=\left[\begin{array}{ll}
3 & 2 \\
2 & 2
\end{array}\right], \quad H_{w_{0}}=\left[\begin{array}{ll}
2 & 1 \\
1 & 2
\end{array}\right], \quad H_{w_{1}}=\left[\begin{array}{ll}
3 & 1 \\
1 & 3
\end{array}\right], \\
h(t)=0.1+0.7 \sin ^{2} t \quad \text { if } t \in \supset=\bigcup \begin{array}{l}
k \geq 0 \\
h(t)=0 \quad \text { if } t \in R^{+} \backslash \supset .
\end{array}
\end{gathered}
$$


Note that $h(t)$ is non-differentiable; therefore, the stability criteria proposed in $[5-8,12,15-$ 26] are not applicable to this system. Given $\alpha=0.3, h_{0}=0.1, h_{1}=0.8$, by using the Matlab LMI toolbox, we can solve for $P, U, G_{0}, G_{1}, H_{0}, H_{1}, D_{0}$, and $D_{1}$ which satisfy the conditions (3.2)-(3.4) in Theorem 3.1. A set of solutions are $\epsilon_{1}=0.9, \epsilon_{2}=0.8, \epsilon_{3}=0.7$,

$$
\begin{aligned}
P=\left[\begin{array}{cc}
0.7832 & -0.0213 \\
-0.0213 & 0.0011
\end{array}\right], & U=\left[\begin{array}{cc}
0.1297 & -0.0019 \\
-0.0019 & 0.0197
\end{array}\right], \\
G_{0}=\left[\begin{array}{ll}
0.1795 & 0.0137 \\
0.0137 & 0.2211
\end{array}\right], & G_{1}=\left[\begin{array}{ll}
1.2197 & 0.9648 \\
0.9648 & 0.7391
\end{array}\right], \\
H_{0}=\left[\begin{array}{cc}
0.8931 & 0.1183 \\
0.1183 & 0.7197
\end{array}\right], & H_{1}=\left[\begin{array}{cc}
0.6851 & 0.1297 \\
0.1297 & 0.5726
\end{array}\right], \\
D_{0}=\left[\begin{array}{cc}
0.1397 & 0 \\
0 & 0.2278
\end{array}\right], & D_{1}=\left[\begin{array}{cc}
0.6812 & 0 \\
0 & 0.6813
\end{array}\right] .
\end{aligned}
$$

Then

$$
u(t)=-0.7314 x_{1}(t)-0.0196 x_{2}(t), \quad t \geq 0,
$$

is a guaranteed cost control law and the cost given by

$$
J^{*}=24.3219\|\phi\|^{2} \text {. }
$$

Moreover, the solution $x(t, \phi)$ of the system satisfies

$$
\|x(t, \phi)\| \leq 12.3690 e^{-0.3 t}\|\phi\|, \quad \forall t \geq 0 .
$$

The exponential convergence dynamics of the network (2.1) are shown in Figure 2.

\section{Conclusions}

In this paper, the problem of guaranteed cost control for uncertain neural networks with interval nondifferentiable time-varying delay has been studied. A nonlinear quadratic cost function is considered as a performance measure for the closed-loop system. The stabilizing controllers to be designed must satisfy some exponential stability constraints on the closedloop poles. By constructing a set of time-varying Lyapunov-Krasovskii functionals combined with Newton-Leibniz formula, a memoryless state feedback guaranteed cost controller design has been presented, and sufficient conditions for the existence of the guaranteed cost state-feedback for the system have been derived in terms of LMIs. 




Figure 2: The simulation of the solutions $x_{1}(t)$ and $x_{2}(t)$ with the initial condition $\phi(t)=[1-1]^{T}, t \in$ $[-1,0.8]$.

\section{Acknowledgments}

This work was supported by the Thai Research Fund Grant, the Higher Education Commission, and Faculty of Science, Maejo University, Thailand. The second author is supported by the Center of Excellence in Mathematics, Thailand, and Commission for Higher Education, Thailand. The authors thank anonymous reviewers for valuable comments and suggestions, which allowed us to improve the paper.

\section{References}

[1] J. J. Hopfield, "Neural networks and physical systems with emergent collective computational abilities," Proceedings of the National Academy of Sciences of the United States of America, vol. 79, no. 8, pp. 2554-2558, 1982.

[2] G. Kevin, An Introduction to Neural Networks, CRC Press, 1997.

[3] M. Wu, Y. He, and J.-H. She, Stability Analysis and Robust Control of Time-Delay Systems, Springer, 2010.

[4] S. Arik, "An improved global stability result for delayed cellular neural networks," IEEE Transactions on Circuits and Systems I, vol. 49, no. 8, pp. 1211-1214, 2002.

[5] K. Ratchagit, "Asymptotic stability of delay-difference system of Hopfield neural networks via matrix inequalities and application," International Journal of Neural Systems, vol. 17, pp. 425-430, 2007.

[6] Y. He, Q.-G. Wang, and M. Wu, "LMI-based stability criteria for neural networks with multiple timevarying delays," Physica D, vol. 212, no. 1-2, pp. 126-136, 2005.

[7] O. M. Kwon and J. H. Park, "Exponential stability analysis for uncertain neural networks with interval time-varying delays," Applied Mathematics and Computation, vol. 212, no. 2, pp. 530-541, 2009.

[8] V. N. Phat and H. Trinh, "Exponential stabilization of neural networks with various activation functions and mixed time-varying delays," IEEE Transactions on Neural Networks, vol. 21, pp. 11801185, 2010.

[9] W.-H. Chen, Z.-H. Guan, and X. Lu, “Delay-dependent output feedback guaranteed cost control for uncertain time-delay systems," Automatica, vol. 40, no. 7, pp. 1263-1268, 2004.

[10] M. N. Parlakçí, "Robust delay-dependent guaranteed cost controller design for uncertain neutral systems," Applied Mathematics and Computation, vol. 215, no. 8, pp. 2936-2949, 2009. 
[11] J. H. Park and O. Kwon, "On guaranteed cost control of neutral systems by retarded integral state feedback," Applied Mathematics and Computation, vol. 165, no. 2, pp. 393-404, 2005.

[12] J. H. Park and K. Choi, "Guaranteed cost control for uncertain nonlinear neutral systems via memory state feedback," Chaos, Solitons and Fractals, vol. 24, no. 1, pp. 183-190, 2005.

[13] J. H. Park and O. M. Kwon, "Guaranteed cost control of time-delay chaotic systems," Chaos, Solitons and Fractals, vol. 27, no. 4, pp. 1011-1018, 2006.

[14] J. H. Park, "Dynamic output guaranteed cost controller for neutral systems with input delay," Chaos, Solitons and Fractals, vol. 23, no. 5, pp. 1819-1828, 2005.

[15] J. H. Park, “Delay-dependent criterion for guaranteed cost control of neutral delay systems," Journal of Optimization Theory and Applications, vol. 124, no. 2, pp. 491-502, 2005.

[16] J. H. Park, "A novel criterion for global asymptotic stability of BAM neural networks with time delays," Chaos, Solitons and Fractals, vol. 29, no. 2, pp. 446-453, 2006.

[17] J. H. Park, "On global stability criterion for neural networks with discrete and distributed delays," Chaos, Solitons and Fractals, vol. 30, no. 4, pp. 897-902, 2006.

[18] H. He, L. Yan, and J. Tu, “Guaranteed cost stabilization of time-varying delay cellular neural networks via Riccati inequality approach," Neural Processing Letters, vol. 35, pp. 151-158, 2012.

[19] J. Tu and H. He, "Guaranteed cost synchronization of chaotic cellular neural networks with timevarying delay," Neural Computation, vol. 24, no. 1, pp. 217-233, 2012.

[20] J. Tu, H. He1, and P. Xiong, "Guaranteed cost synchronous control of time-varying delay cellular neural networks," Neural Computing and Applications.

[21] H. He and J. Tu, "Algebraic condition of synchronization for multiple time-delayed chaotic Hopfield neural networks," Neural Computing and Applications, vol. 19, pp. 543-548, 2010.

[22] H. He, J. Tu, and P. Xiong, " $L_{r}$-synchronization and adaptive synchronization of a class of chaotic Lurie systems under perturbations," Journal of the Franklin Institute, vol. 348, no. 9, pp. 2257-2269, 2011.

[23] E. Fridman and Y. Orlov, "Exponential stability of linear distributed parameter systems with timevarying delays," Automatica, vol. 45, no. 1, pp. 194-201, 2009.

[24] S. Xu and J. Lam, "A survey of linear matrix inequality techniques in stability analysis of delay systems," International Journal of Systems Science, vol. 39, no. 12, pp. 1095-1113, 2008.

[25] J.-S. Xie, B.-Q. Fan, Y. S. Lee, and J. Yang, "Guaranteed cost controller design of networked control systems with state delay," Acta Automatica Sinica, vol. 33, no. 2, pp. 170-174, 2007.

[26] L. Yu and F. Gao, "Optimal guaranteed cost control of discrete-time uncertain systems with both state and input delays," Journal of the Franklin Institute, vol. 338, no. 1, pp. 101-110, 2001.

[27] S. Boyd, L. El Ghaoui, E. Feron, and V. Balakrishnan, Linear Matrix Inequalities in System and Control Theory, vol. 15, SIAM, Philadelphia, Pa, USA, 1994.

[28] K. Gu, V. Kharitonov, and J. Chen, Stability of Time-delay Systems, Birkhauser, Berlin, Germany, 2003. 


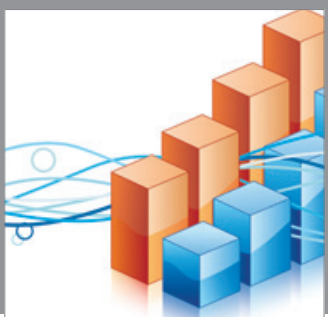

Advances in

Operations Research

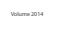

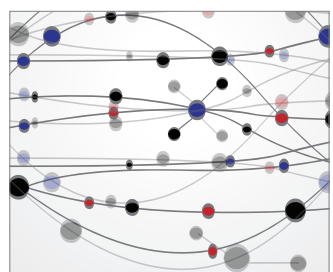

\section{The Scientific} World Journal
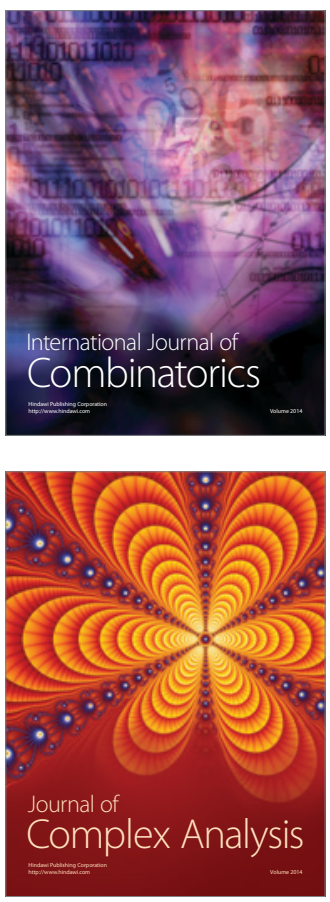

International Journal of

Mathematics and

Mathematical

Sciences
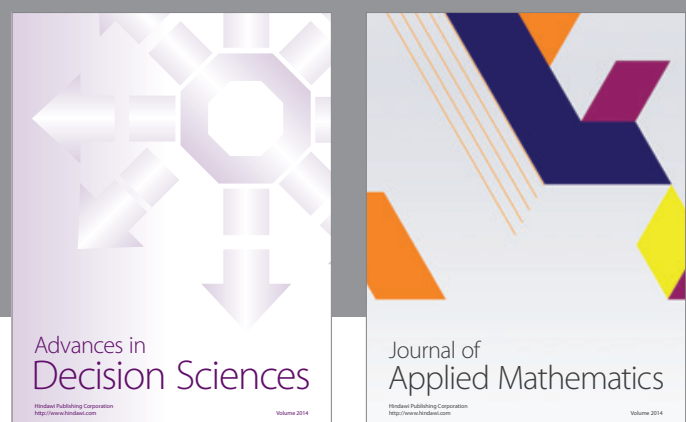

Journal of

Applied Mathematics
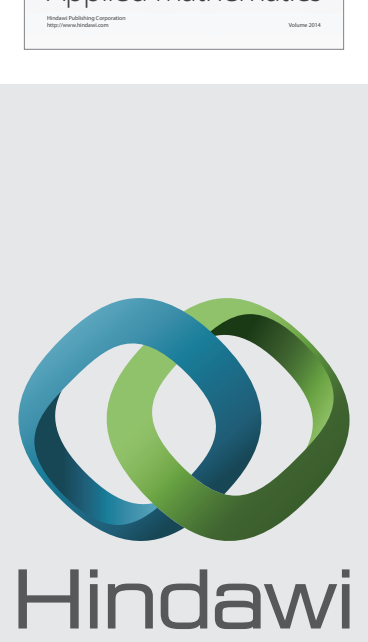

Submit your manuscripts at http://www.hindawi.com
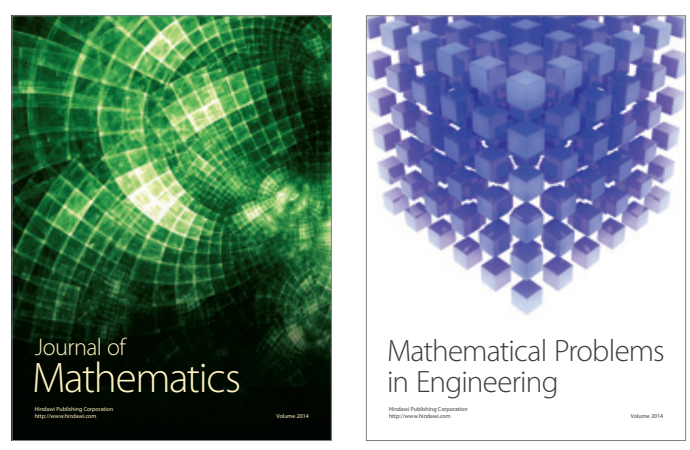

Mathematical Problems in Engineering
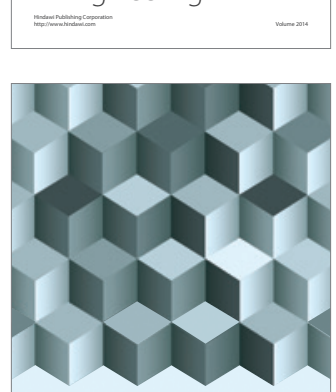

Journal of

Function Spaces
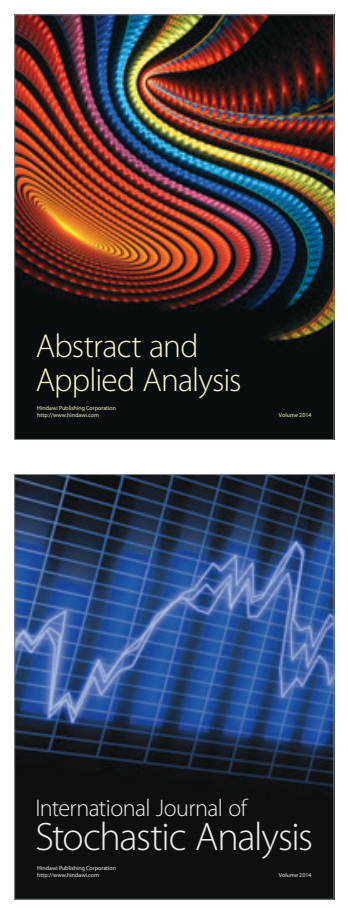

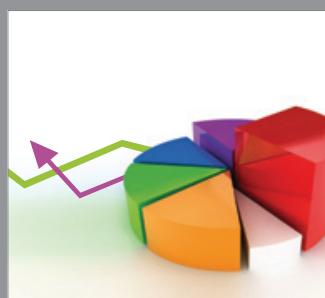

ournal of

Probability and Statistics

Promensencen
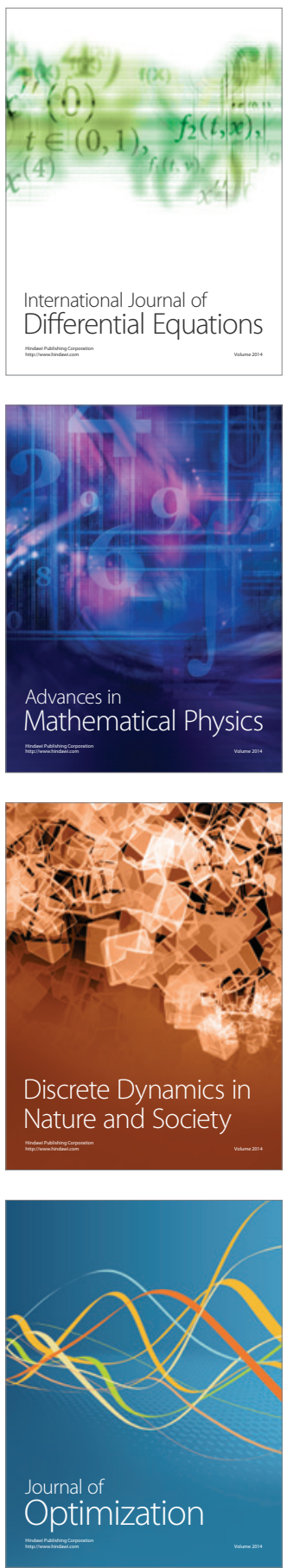